\title{
Patient-rated versus proxy-rated cognitive and functional measures in older adults
}

\author{
Molly Howland' \\ Kevin C Allan' \\ Caitlin E Carlton' \\ Curtis Tatsuoka ${ }^{2-4}$ \\ Kathleen A Smyth ${ }^{3}$ \\ Martha Sajatovic ${ }^{1,2,4,5}$ \\ 'Case Western Reserve University \\ School of Medicine, ${ }^{2}$ Neurological \\ and Behavioral Outcomes Center, \\ University Hospitals Case Medical \\ Center, ${ }^{3}$ Department of Epidemiology \\ and Biostatistics, ${ }^{4}$ Department of \\ Neurology, ${ }^{5}$ Department of Psychiatry, \\ Case Western Reserve University \\ School of Medicine, Cleveland, \\ OH, USA
}

This article was published in the following Dove Press journal:

Patient Related Outcome Measures

15 March 2017

Number of times this article has been viewed

Objectives: Patients with cognitive impairment may have difficulty reporting their functional and cognitive abilities, which are important clinical outcomes. Health care proxies may be able to corroborate patient self-reports. Several studies reported discrepancy between patient and proxy ratings, though the literature is sparse on changes over time of these ratings. Our goals in this 12-month study were to compare patient and proxy reports on functioning, cognition, and everyday executive function, and to further elucidate correlates of patient-proxy discrepancy.

Methods: This was a prospective cohort study of individuals older than 70 years who ranged from having no cognitive impairment to having moderate dementia who had a proxy available to complete instruments at baseline $(\mathrm{N}=76)$. Measurements included Alzheimer's Disease Cooperative Study-Activities of Daily Living Inventory (ADCS-ADLI), Neuro-QOL Executive Function, PROMIS Applied Cognition (PROMIS-Cog), Mini-Mental State Examination (MMSE), and Geriatric Depression Scale.

Results: Patient- and proxy-rated ADCS-ADLI were correlated at baseline and at 1-year follow-up. Patient and proxy ratings were discrepant on Neuro-QOL Executive Function and PROMIS-Cog. Greater patient-proxy discrepancy on PROMIS-Cog was associated with younger age and less depression, and greater patient-proxy discrepancy on Neuro-QOL Executive Function was associated with less depression and worse cognitive impairment. Patient-proxy discrepancy increased over time for everyday executive function. Changes in proxy-rated but not patient-rated ADCS-ADLI correlated with MMSE changes.

Conclusion: Patients and proxies generally agree in reporting on activities of daily living. Patient and proxy reports differ in their respective evaluation of cognitive functioning and everyday executive function. Ratings from both sources may be preferred for these two domains, though studies using gold standard measures are necessary. It is important that clinicians are aware of the differences between patient and proxy perspective to create an accurate clinical picture and guide treatment.

Keywords: agreement, activities of daily living, dementia, executive function, caregiver

\section{Introduction}

Alzheimer's disease (AD) is associated with cognitive and functional decline, eroding patients' quality of life (QOL). ${ }^{1}$ In a consensus conference in 1997, AD experts declared the primary goals of $\mathrm{AD}$ treatment to be improved QOL and functioning through elevation of mood, behavior, and cognitive status. ${ }^{2}$ Given the importance of functioning in determining care needs such as social supports and evaluating intervention efficacy, clinicians must be able to measure these functional components as clinical endpoints,

Correspondence: Molly Howland Neurological and Behavioral Outcomes Center, University Hospitals Case Medical Center, 10524 Euclid Avenue, Cleveland, OH 44I06, USA

$\mathrm{Tel}+\mathrm{I} 2168442808$

Fax + I 2168442742

Email molly.howland@case.edu 
as was recommended in 2010 by the US National Institutes of Health (NIH) in their Consensus Development Conference Statement on Preventing Alzheimer's Disease and Cognitive Decline. ${ }^{3}$ Executive function in particular is one of the first abilities to decline and may even begin to decline before the $\mathrm{AD}$ diagnosis, necessitating assessment of this construct in older adults. ${ }^{4}$

Assessment options for clinicians include performancebased measures of cognitive ability - such as the Mini-Mental State Examination (MMSE) - and functional ability - such as the Direct Assessment of Functional Status. These performance-based measures are objective but involve simplified tasks performed in the experimental setting only and, therefore, may not reflect real-world tasks. ${ }^{5,6}$ Thus, instead of these objective measures, clinicians often rely on subjective reports by patients or, given the importance of social support in AD care, the patient's caregiver. In some cases, self-reports may be inaccurate because of AD-related cognitive impairment. ${ }^{7}$ Caregivers can serve as health care proxies who may be able to more accurately report patients' abilities.

Other research groups have studied the relationships between patient-rated, proxy-rated, and performance-based measures. Prior studies have consistently found that patients rate their QOL higher compared to proxy ratings, ${ }^{8-13}$ while satisfactory correlations have been found between patientand proxy-rated ADL measures. ${ }^{14,15}$ Several studies have identified sources of patient-proxy discrepancy, which include severity of dementia, ${ }^{9,14}$ relationship between patient and proxy; ${ }^{5,12,14,16}$ depressive symptoms, ${ }^{13}$ and the degree to which the abilities tested are directly observable by the proxy. ${ }^{12,14,15}$ However, the association between these determinants and patient-proxy discrepancy has been inconsistent across studies. Additionally, literature is sparse on the discrepancy between patient- and proxy-reported subjective cognitive function and changes over time in patient-proxy discrepancy in any domain.

This analysis from a larger prospective NIH-funded study compared patient-rated and proxy-rated measures of functioning at baseline and at 12-month follow-up using the Alzheimer's Disease Cooperative Study-Activities of Daily Living Inventory (ADCS-ADLI), the Executive Function form of the Quality of Life in Neurological Disorders (Neuro-QOL Executive Function) scale, and the Applied Cognition form from the Patient-Reported Outcomes Measurement Information System (PROMIS $®$ Applied Cognition or PROMIS-Cog). Our objectives were to examine patient-proxy discrepancy across several domains both at baseline and at 12 months. We aimed to elucidate the correlates of these disparities, especially for the little-studied QOL related to executive function, or everyday executive function, construct. Since the evidence indicates higher patient-proxy agreement for directly observable functioning than for mental processes, ${ }^{12,14,15}$ we hypothesized that we would see relatively high agreement on the ADCS-ADLI and low agreement on the Neuro-QOL Executive Function and PROMIS-Cog.

\section{Methods}

\section{Sample}

A parent cohort of 304 participants was recruited between May 2011 and September 2013 from neurology and geriatric clinics and the community in Ohio. A small percentage of participants were recruited during a short stay at an in-patient geropsychiatric unit of an academic medical center. All participants were asked if they could identify a family member or close friend who could serve as a proxy to accompany the participant to their visits and complete proxy-rated versions of assessments. Twenty-five percent of participants $(\mathrm{N}=76)$ had a proxy available to complete instruments at baseline. We analyzed only this subsample in our study. After 12 months, during which no interventions were implemented, participants completed assessments again.

Inclusion criteria were broad with minimal exclusion criteria to increase external validity. Participants were deemed eligible if 1) 70 years old or older; 2) MMSE score was $>16$ and patient self-identified as without cognitive impairment, having diagnosis of mild cognitive impairment (MCI) or having diagnosis of mild to moderate dementia; 3) able to read and speak English; and 4) able to provide informed consent at the time of the initial baseline interview. Individuals were excluded if 1) life expectancy was $<12$ months; 2) planning to be placed in a nursing home or move from the greater Cleveland area within 12 months; 3 ) actively abusing or dependent on a substance; or 4) suffering from an uncontrolled mental disorder that would limit the individual's ability to complete the study questionnaires. The team attempted to assess proxies who were in contact with patients at least 2 days per week but ended up with a small proportion of proxies who visited with the patient less frequently (Table 1). Proxies were given a separate informed consent document. Written informed consent was obtained from all participants and proxies.

All study procedures were deemed consistent with the Helsinki Declaration of 1975 (as revised in 1983) and this study was approved by the Institutional Review Board of University Hospitals of Cleveland, OH, USA. 
Table I Patient and proxy demographic information

\begin{tabular}{|c|c|c|c|}
\hline Characteristics & $\begin{array}{l}\text { PROMIS Applied Cognition } \\
(\mathrm{N}=74)\end{array}$ & $\begin{array}{l}\text { Neuro-QOL Executive Function } \\
(\mathrm{N}=59)\end{array}$ & ADCS-ADLI $(\mathrm{N}=48)$ \\
\hline Age (years) & $80.3(6.6)$ & $80.7(6.8)$ & $81.1(6.6)$ \\
\hline \multicolumn{4}{|l|}{ Gender } \\
\hline Female & $67.6(50)$ & $64.4(38)$ & $66.7(32)$ \\
\hline Male & $32.4(24)$ & $35.6(21)$ & $33.3(16)$ \\
\hline Education level & $8.1(6)$ & $8.5(5)$ & $6.3(3)$ \\
\hline Less than high school degree & $24.3(18)$ & $20.3(12)$ & $20.8(10)$ \\
\hline High school degree/some college & $18.9(14)$ & $20.3(12)$ & $25.0(12)$ \\
\hline College degree or above & $48.7(36)$ & $50.8(30)$ & $47.9(23)$ \\
\hline \multicolumn{4}{|l|}{ Race } \\
\hline Caucasian & $78.4(58)$ & $79.7(47)$ & $79.2(38)$ \\
\hline African-American & $21.6(16)$ & $20.3(12)$ & $20.8(10)$ \\
\hline Number of medical conditions & $4.0(2.2)$ & $4.0(2.0)$ & $4.0(1.7)$ \\
\hline MMSE score & $26.1(3.5)$ & $26.4(3.3)$ & $26.3(3.5)$ \\
\hline \multicolumn{4}{|l|}{ MMSE category } \\
\hline Normal & $10.6(7)$ & $11.8(6)$ & $13.6(6)$ \\
\hline Mild cognitive impairment & $33.3(22)$ & $33.3(17)$ & $29.5(13)$ \\
\hline Dementia & $56.1(37)$ & $54.9(28)$ & $56.8(25)$ \\
\hline GDS score & $4.9(4.6)$ & $5.2(4.8)$ & $5.6(5.0)$ \\
\hline \multicolumn{4}{|l|}{ GDS category } \\
\hline Normal & $83.1(59)$ & $80.7(46)$ & $76.1(35)$ \\
\hline Mild & $16.9(12)$ & $19.3(11)$ & $23.9(11)$ \\
\hline Severe & $0.0(0)$ & $0.0(0)$ & $0.0(0)$ \\
\hline \multicolumn{4}{|l|}{ Proxy relationship (\%) } \\
\hline Spouse & $46.5(33)$ & $44.8(26)$ & $45.7(2 \mathrm{I})$ \\
\hline Child & $33.8(24)$ & $36.2(21)$ & $39.1(18)$ \\
\hline Other relative & $9.9(7)$ & $10.3(6)$ & $8.7(4)$ \\
\hline Friend & $7.0(5)$ & $6.9(4)$ & $6.5(3)$ \\
\hline Other & $2.8(2)$ & $1.7(\mathrm{I})$ & $0.0(0)$ \\
\hline \multicolumn{4}{|l|}{ Frequency of contact (\%) } \\
\hline Lives with participant & $61.4(43)$ & $56.1(32)$ & $60.0(27)$ \\
\hline At least once a day & $20.0(14)$ & $24.6(14)$ & $26.7(12)$ \\
\hline At least once a week & I7.I (12) & $17.5(10)$ & $13.3(6)$ \\
\hline At least once a month & $\mathrm{I} .4(\mathrm{I})$ & $1.8(\mathrm{I})$ & $0.0(0)$ \\
\hline
\end{tabular}

Note: Data shown as percentage (frequency) or mean (SD).

Abbreviations: ADCS-ADLI, Alzheimer's Disease Cooperative Study-Activities of Daily Living Inventory; GDS, Geriatric Depression Scale; MMSE, Mini-Mental State Examination; PROMIS, Patient-Reported Outcomes Measurement Information System; QOL, quality of life; SD, standard deviation.

\section{Measures}

All study measures were conducted at baseline and at 1-year follow-up. Proxy measures substituted the pronoun "I" with "the research participant."

1. ADCS-ADLI:ADCS-ADLI consists of 23 items designed to evaluate functional performance in individuals with $\mathrm{MCI}$ and $\mathrm{AD}$ for clinical trials. In a multicenter developmental protocol, ADCS-ADLI showed good test-retest reliability, correlated with MMSE scores on AD patients, and demonstrated decline in performance from baseline to 12 months. ${ }^{17}$ Higher scores indicate better functionality. Example questions include "In the past 3 weeks did you select your first set of clothes for the day? (Yes/No/ Don't know). If yes, which best describes how you usually perform: (with physical help/with supervision/without supervision of help/does not apply)."
2. PROMIS-Cog: PROMIS-Cog is a 16-item instrument that evaluates impressions of cognitive function in the past week in areas such as mental acuity, concentration, and memory ${ }^{18}$ Prior studies have demonstrated an internal consistency of $>0.90 .{ }^{19}$ Higher scores denote better cognitive functioning. Example questions include "My thinking has been as fast as usual," and "I have been able to remember things as easily as usual without extra effort."

3. Neuro-QOL Executive Function: Neuro-QOL was developed through a multisite project to be a clinically relevant and psychometrically robust health-related QOL assessment tool for adults and children. The executive function form contains items assessing cognitive functioning in the context of activities of daily living. ${ }^{20}$ The Executive Function item bank contains 13 items that 
ask the respondent to indicate on a 5-point scale (none, a little, somewhat, a lot, cannot do) how much difficulty they currently have with certain everyday cognitive abilities. Higher scores indicate higher executive functioning abilities. An example question is "How much difficulty do you currently have planning an activity several days in advance (e.g., a meal, a trip, or a visit to friends)."

4. Geriatric Depression Scale (GDS): The GDS is a 30-item scale that assesses depression in older individuals. The measure involves yes/no responses, which require less cognitive ability compared with Likert scales. ${ }^{21}$ The GDS has shown strong psychometric properties such as robust internal consistency (Cronbach's $\alpha=0.91$ ), splithalf reliability of 0.94 , and a test-retest correlation of 0.85 over 1 month. ${ }^{22}$ Higher scores indicate higher levels of depression, with a cutoff for "mildly depressed" of 15 and a cutoff for "severely depressed" of $23 .{ }^{21}$

5. Mini-Mental State Examination (MMSE): The MMSE is a 12-item cognitive screening tool with 26 question subparts that evaluates an individual's orientation to time and place, registration of words, attention and calculation, recall of words, and visual construction. ${ }^{23}$

\section{Data analysis}

\section{Descriptive analysis}

For a cohort of participants who completed each measure themselves and also had a proxy who completed the measure, we used descriptive statistics to characterize participant demographics including age, gender, education level, race, number of medical conditions, MMSE score and cognitive category (according to the optimized cutoffs suggested by Tariq et al, 2006), ${ }^{24}$ and GDS depression score and status. Optimized MMSE cutoffs were $<28.5$ for MCI and <26.5 for dementia for participants who did not complete high school and 1 point higher than these values for participants who completed high school. We also characterized proxy demographics such as relationship to the participant and frequency of contact with the participant (Table 1). To compare the participants with proxies available and parent cohort participants without proxies, we performed two-sided, independent-samples $t$-tests (assuming unequal variance when appropriate) or chi-square tests for the above demographic variables and the three measures of interest.

To determine the relationships among the constructs assessed by PROMIS-Cog, Neuro-QOL Executive Function, and ADCS-ADLI, we performed Spearman correlations between these measures at baseline. Additionally, to determine the magnitude of change in objective cognitive performance, we calculated the mean and standard deviation (SD) for 1-year change in MMSE.

\section{Magnitude of patient-proxy discrepancy}

We determined the mean and SD for each measure at baseline for both participants and proxies. We performed Spearman correlations on these means and bidirectional paired-samples $t$-tests.

Because of the low overlap between proxies who completed PROMIS-Cog at baseline and at 1 year $(\mathrm{N}=9)$, we decided to exclude this measure from the follow-up analysis. To conduct the follow-up analysis, we determined the mean and SD for 12-month changes (calculated as 12-month time point minus baseline) in patient and proxy scores on NeuroQOL Executive Function and ADCS-ADLI. For each measure, we performed Spearman correlations between patient and proxy changes in scores. We performed a one-sample $t$-test for the change over time in patient-proxy discrepancy.

\section{Correlates of patient-proxy discrepancy}

To identify correlates of patient-proxy discrepancy, we correlated the difference between patient and proxy scores at baseline (calculated as patient score minus proxy score) with age, MMSE, and GDS. With discrepancy as the dependent variable, we performed independent-samples $t$-tests for gender and one-way analyses of variance (ANOVAs) with Tukey's post hoc tests for MMSE cognitive category, proxy relation, and proxy frequency of contact with the participant. Next we fit joint regression models for each measure with gender, proxy relation, proxy frequency of contact, age, MMSE total score, and GDS as initial variables. To simplify these models, we eliminated the gender, proxy relation, and proxy frequency of contact variables since these variables were not significant in the original regression models. We generated scatter plots for scores on the measures versus covariates such as age, MMSE, and GDS.

\section{Validation of patient and proxy ratings}

To determine which measures best matched changes in objective cognition while assessing the role of depression in these changes over time, we correlated changes in patient and proxy scores on Neuro-QOL Executive Function and ADCS-ADLI with changes in MMSE and GDS. We then jointly fit changes in MMSE and GDS as covariates in respective regressions for change in patient-rated or proxy-rated measures.

All analyses were conducted using Statistical Package for the Social Sciences (SPSS) Version 22. 


\section{Results}

\section{Descriptive analysis}

Most of our patients were nondepressed, white, educated women with a mean age of $\sim 80$ and a mean MMSE score of $\sim 26$, categorizing most participants as having dementia (Table 1). Spouses were the most common type of health care proxy, while adult children were the second most common type. The remaining proxies were other relatives, friends, or "other." Most proxies lived with patients, though some visited with the patient "at least once a day" or "at least once a week," and one proxy visited only "at least once a month." Compared with participants in the parent cohort with no proxy available, our subsample was older ( 80.2 years versus 77.7 years, $t=3.18, p=0.006$ ), more cognitively impaired (MMSE score of 26.1 versus 27.9, $t=-5.26, p<0.001$ ), and scored lower on Neuro-QOL Executive Function (51.7 versus 55.4, $t=-3.23, p=0.009)$ and ACDS-ADLI (36.0 versus $41.0, t=-6.38, p<0.001)$. Gender, education, race, number of medical conditions, GDS score, and PROMIS-Cog score did not differ significantly between our subsample and the parent cohort participants with no proxy.

To determine whether our three measures were assessing similar constructs, we performed Spearman correlations that showed that PROMIS-Cog was moderately correlated with both ADCS-ADLI and Neuro-QOL Executive Function $(\rho=0.40, p=0.006 ; 0.52, p<0.001)$. ADCS-ADLI and NeuroQOL Executive Function were strongly correlated $(\rho=0.72$, $p<0.001)$. In the follow-up analysis, mean 12-month change in MMSE was -1.9 (SD 3.6).

\section{Magnitude of patient-proxy discrepancy}

Patient and proxy baseline scores are listed in Table 2. Patient and proxy ratings were mildly to moderately correlated for PROMIS-Cog and Neuro-QOL Executive Function
(Table 3). Patient and proxy ratings were strongly correlated for ADCS-ADLI. Paired-samples $t$-tests identified significant differences between patient and proxy ratings for PROMIS-Cog and Neuro-QOL Executive Function but not for ADCS-ADLI (Table 3).

Mean change in participant-rated Neuro-QOL Executive Function was 0.9 (SD 6.7), while mean change in proxyrated Neuro-QOL Executive Function was -2.7 (SD 8.0), producing an increase over time in the difference between patient and proxy ratings of 3.7 (SD 9.5, $t=2.49, p=0.018$ ). Change in proxy and participant Neuro-QOL Executive Function differences did not correlate significantly $(\rho=0.18$, $p=0.262, \mathrm{~N}=41)$. Mean change for participant-rated ADCSADLI was -2.6 (SD 8.0), and mean change for proxy-rated ADCS-ADLI was -4.7 (SD 6.3), corresponding to a mean increase in discrepancy of 2.1 (SD 6.9), which did not reach statistical significance $(t=1.87, p=0.07)$. Change in proxy and participant ADCS-ADLI moderately correlated $(\rho=0.55$, $p<0.001, \mathrm{~N}=38$ ).

\section{Correlates of patient-proxy discrepancy}

Ratings given by patients and proxies for Neuro-QOL Executive Function were more divergent when the patient had poorer cognitive function per MMSE and lower depression levels (Table 4). A higher patient-proxy discrepancy for PROMIS-Cog was correlated with lower depression levels, but no significant relationship was found with MMSE. Age did not correlate with any measure in these initial Spearman correlations. Independent-samples $t$-tests or ANOVAs performed for gender, MMSE cognitive category, proxy relation, and proxy frequency of contact were not significant.

Linear regression performed for patient-proxy discrepancy using the simplified models (including age, MMSE, and GDS as variables) indicated that age was significant

Table 2 Mean patient and proxy ratings at baseline

\begin{tabular}{llll}
\hline & $\begin{array}{l}\text { PROMIS Applied } \\
\text { Cognition }(\mathbf{N}=\mathbf{7 4})\end{array}$ & $\begin{array}{l}\text { Neuro-QOL Executive } \\
\text { Function (N=59) }\end{array}$ & ADCS-ADLI (N=48) \\
\hline Patient rating, mean (SD) & $57.0(I I .4)$ & $51.5(10.8)$ & $35.9(8.6)$ \\
Proxy rating, mean (SD) & $51.1(12.8)$ & $46.0(13.8)$ & $34.3(9.5)$ \\
\hline
\end{tabular}

Abbreviations: ADCS-ADLI, Alzheimer's Disease Cooperative Study-Activities of Daily Living Inventory; PROMIS, Patient-Reported Outcomes Measurement Information System; QOL, quality of life; SD, standard deviation.

Table 3 Spearman correlations and t-tests for patient versus proxy ratings at baseline

\begin{tabular}{llll}
\hline & $\begin{array}{l}\text { PROMIS Applied } \\
\text { Cognition }(\mathbf{N}=\mathbf{7 4})\end{array}$ & $\begin{array}{l}\text { Neuro-QOL Executive } \\
\text { Function }(\mathbf{N}=59)\end{array}$ & ADCS-ADLI(N=48) \\
\hline Spearman correlation coefficient, $p$-value & $0.45,<0.00 I^{* *}$ & $0.35,0.007^{*}$ & $0.81,<0.00 I^{* *}$ \\
$t$-value, $p$-value & $4.05,<0.00 I^{* *}$ & $3.08,0.004^{*}$ & $1.94,0.060$ \\
\hline
\end{tabular}

Notes: *Significant at $p<0.05 ; * *$ significant at $p<0.01$.

Abbreviations: ADCS-ADLI, Alzheimer's Disease Cooperative Study-Activities of Daily Living Inventory; PROMIS, Patient-Reported Outcomes Measurement Information System; QOL, quality of life. 
Table 4 Spearman correlations of patient-proxy discrepancy with age, MMSE, and GDS

\begin{tabular}{llll}
\hline & PROMIS Applied Cognition & Neuro-QOL Executive Function & ADCS-ADLI \\
\hline Age, $p$-value & $-0.22,0.059, \mathrm{~N}=74$ & $-0.08,0.56, \mathrm{~N}=59$ & $-0.18,0.22, \mathrm{~N}=48$ \\
MMSE, $p$-value & $-0.16,0.19, \mathrm{~N}=74$ & $-0.42,<0.00 I^{* *}, \mathrm{~N}=59$ & $-0.15,0.3 \mathrm{~N}, \mathrm{~N}=48$ \\
GDS, $p$-value & $-0.28,0.018^{*}, \mathrm{~N}=71$ & $-0.40,0.003^{*}, \mathrm{~N}=57$ & $-0.17,0.26, \mathrm{~N}=46$ \\
\hline
\end{tabular}

Notes: $\mathrm{N}$ values represent number of patient-proxy pairs. *Significant at $p<0.05$; **significant at $p<0.01$.

Abbreviations: ADCS-ADLI, Alzheimer's Disease Cooperative Study-Activities of Daily Living Inventory; GDS, Geriatric Depression Scale; MMSE, Mini-Mental State Examination; PROMIS, Patient-Reported Outcomes Measurement Information System; QOL, quality of life.

$(\mathrm{B}=-0.54, p=0.015)$ for PROMIS-Cog, indicating that patients and proxies agreed more at older patient ages. GDS was no longer significant $(\mathrm{B}=-0.53, p=0.097)$ for PROMIS$\mathrm{Cog}$. The simplified regression model for Neuro-QOL Executive Function revealed that both MMSE $(\mathrm{B}=-1.52, p=0.003)$ and GDS $(\mathrm{B}=-0.81, p=0.021)$ were significant, reflecting greater patient-proxy discrepancy when the patient had poorer cognitive function and less depression. At lower GDS scores, scores on both Neuro-QOL Executive Function and PROMIS-Cog tended toward the maximum score. None of the variables were significant for ADCS-ADLI.

\section{Validation of patient and proxy ratings}

Neither proxy nor participant Neuro-QOL Executive Function changes correlated with changes in MMSE or GDS. Change in proxy ADCS-ADLI score correlated with change in MMSE ( $\rho=0.31, p=0.042)$ but not change in GDS. Change in participant ADCS-ADLI score did not correlate significantly with change in MMSE or GDS. Linear regression confirmed the association between change in proxy ADCS-ADLI score and change in MMSE $(\mathrm{B}=1.191, p=0.002)$.

\section{Discussion}

This analysis of patient- versus proxy-rated outcomes in older people with varying degrees of cognitive function found that proxies and patients are likely to agree on ADL measures but not on measures of applied cognition or QOL related to executive function. Though prior studies have looked at ADL, cognitive, and QOL measures separately, we are the first to directly compare patient and proxy ratings on all three. QOL related to executive function, or everyday executive function, is a construct that is related to but distinct from general QOL. For this construct, we are the first to identify the correlates of patient-proxy discrepancy and assess these ratings over time. Patient-proxy discrepancy increased over 12 months for QOL related to executive function. Greater patient-proxy discrepancy on Neuro-QOL Executive Function was associated with less depression and worse cognitive impairment. To our knowledge, we are the first to report that greater patient-proxy discrepancy on an applied cognition measure was associated with less depression and younger age.

\section{Magnitude of patient-proxy discrepancy}

Similar to proxies' reports of lower QOL related to executive function, proxies reported significantly greater impairment than patients in the applied cognition domain, perhaps reflecting anosognosia. ${ }^{25}$ Researchers have begun establishing that proxy-reported cognitive impairment may be more correlated than patient-reported cognitive impairment to objective cognitive performance. ${ }^{25}$ However, proxies may lack information about less observable cognitive domains. ${ }^{26}$ "Mutual complaint," or cognitive dysfunction noted by both patients and proxies, is more associated than patient or proxy report with neuropathology ${ }^{27}$ and more predictive of future cognitive decline. ${ }^{28}$ Thus, having applied cognition information from both sources may be preferable to one or the other.

Our 12-month study with multiple instruments completed by both participants and proxies allowed us to compare the trajectory of ADL and QOL related to executive function over time. Consistent with previous cross-sectional findings, patients and proxies are likely to agree on ADL assessments but not on QOL assessments. ${ }^{8,9,11,12-15}$ One previous study identified no correlation between changes over time in patient- and proxy-rated general QOL measures. ${ }^{29}$ One study found that proxy ratings for Neuro-QOL Executive Function were lower than patient ratings in a patient population with stroke; ${ }^{30}$ however, we are the first to evaluate this particular measure's performance over time in a general older population. The patient-proxy discrepancy for QOL related to executive function might be explained by the subjective nature of QOL and executive processes as opposed to the direct observability of ADLs such as cleaning or keeping appointments. ${ }^{12,15,16} \mathrm{~A}$ recent study noted that patient-proxy discrepancy in QOL related to memory was significant beginning in mild dementia, whereas other QOL domains - including "life as a whole" - became significant at moderate levels of dementia. ${ }^{31}$ Since most of our study's participants with dementia had only mild dementia, our results support that discrepancy for QOL related to cognitive processes appears early in dementia. Therefore, it is important that clinicians recognize that proxies may have a more negative perspective than patients of patients' everyday executive function even early in the disease process. 
Proxy ratings of QOL related to executive function were more likely to decline over time than patient ratings, which increased slightly on average. Many previous reports have illustrated that a discrepancy exists between patient- and proxy-rated measures of QOL, but to our knowledge, none have illustrated that the disagreement between patient and proxy assessments increases over time. In line with proxies' lower estimation of patients' QOL and abilities, perhaps proxies also have more pessimistic expectations for the course of the disease. Discrepancy in ADL ratings did not significantly increase over time, though we did observe a trend toward proxy ratings decreasing more over time than patient ratings, supporting results from a 1994 study. ${ }^{7}$ The decline in patient ratings indicates that cognitive impairment does not prevent patients from detecting decline in ADLs.

It has been suggested that the patient's own experiences are crucial to the definition of QOL, especially given that individuals with similar conditions may have different QOL because of their attitudes and beliefs. ${ }^{32}$ Brod et al posited that, though people with mild to moderate dementia may not be aware of cognitive deficit, they are aware of their current emotional states. ${ }^{32}$ These findings in conjunction with the discrepancy between patient and proxy reports of QOL discourage relying on proxy-reported QOL for patients with mild to moderate dementia. However, QOL related to executive function is a related but distinct construct from general QOL that incorporates mental processes and was correlated moderately with PROMIS-Cog. Both patients and proxies may provide valuable reports on QOL related to executive function, similar to applied cognition. Conversely, clinicians may be able to trust either a patient- or proxy-reported ADL measure score by itself.

\section{Correlates of patient-proxy discrepancy}

In this analysis, greater patient depression was associated with greater agreement between patients and proxies on QOL related to executive function and applied cognition. Though higher depression levels have been associated with higher patient-proxy agreement on the MMSE, ${ }^{33}$ we appear to be the first to report these associations for subjective cognition measures. The scores of patients with lower depression levels were clustered near the maximum for both applied cognition and QOL related to executive function. Both applied cognition and QOL have previously been associated with depression. ${ }^{25,34,35}$ Therefore, perhaps as depression levels increase, both patients and proxies become attuned to deficits in cognition and QOL related to executive function. We, thus, confirm the results of Tay et al that greater depression levels correlate with lower patient-rated general QOL to match proxies' estimation of lower QOL. ${ }^{13}$ We further posit that greater depression associates with greater patient-proxy agreement for QOL related to executive function and applied cognition. The positive association between age and patient-proxy agreement on applied cognition may be explained by patients' lower expectations for their cognitive functioning as they age, matching proxies' expectations.

Cognitive impairment on MMSE was significantly associated with greater patient-proxy discrepancy on Neuro-QOL Executive Function. Some studies have failed to demonstrate a relationship between dementia severity and patient versus proxy QOL reports. ${ }^{10,29}$ However, similar to our findings, one study reported that proxy QOL ratings decreased with progressive cognitive decline while patient QOL did not decrease. ${ }^{9}$ Perhaps proxies equate a decline in cognition with a decline in QOL related to executive function, while patients either adopt a different perspective or lack insight into their QOL related to executive function. ${ }^{11} \mathrm{~A}$ recent study found that patient-proxy discrepancy on only certain QOL items - including memory, a component of executive function - increased with decreasing cognitive function, supporting our results. ${ }^{31}$

Additionally, it was interesting that degree of cognitive impairment did not correlate with patient-proxy discrepancy in applied cognition. One might expect a progressive anosognosia - and thus increased patient-proxy discrepancy - with increasing dementia severity. However, factors other than dementia severity can also contribute to anosognosia, ${ }^{36}$ and patient-proxy discrepancy is also related to proxy characteristics. ${ }^{6}$

Other studies have found that more severe dementia correlates to greater patient-proxy discrepancy in ADL measures. ${ }^{14,15}$. However, these studies did not exclude individuals with MMSE scores of 16 . Individuals with very severe dementia may disagree more with their proxies.

We did not identify significant relationships between any domain and proxy relationship or frequency of patient-proxy contact. Data have been inconsistent on the link of proxy relationship and frequency of contact with patient-proxy discrepancy for both QOL and ADL measures., ${ }^{3,10,12,14}$ Huang et al suggested that the quality of the patient-proxy relationship may play a larger role in patient-proxy discrepancy. ${ }^{16}$ While we did not evaluate caregiver burden or depression, these variables may also be important mediators of discrepancy. $1,6,16,37$ 


\section{Validation of patient and proxy ratings}

Though changes in patient and proxy ADL ratings were moderately correlated, only changes in proxy ADL ratings were significantly associated with MMSE changes. This confirms the results of Kiyak and colleagues (1994), who found that family member - but not patient - reports of declines in physical and instrumental ADLs (PADLs and IADLs) correlated with 2-year declines in cognitive abilities measured by formal testing. ${ }^{7}$ Proxies may be more attuned than patients to small changes in patients' instrumental ADLs - which the ADCS-ADLI is more heavily weighted toward - than physical ADLs. ${ }^{14,15,38}$ In AD, the first abilities to decline are often instrumental and include balancing a checkbook or going shopping, while physical abilities such as bathing, dressing, and walking decline later. In milder cognitive impairment, patients may not be as aware of subtle instrumental deficits as proxies are. ${ }^{34}$ Indeed, weaker patient-proxy agreement has been found for IADLs than PADLs. ${ }^{38}$ Our sample on average had mild dementia at baseline and small decreases in cognition over time; participants may not have yet recognized deficits in ADLs. Thus, it may be wise to weight proxy ratings more heavily when assessing decline in IADLs over time.

A few studies have explored the usefulness of combined patient-proxy measures. Gifford and colleagues (2015) used a "mutual cognitive complaint" measure, which predicted future cognitive decline better than patient-only or proxy-only reports. ${ }^{28}$ Additionally, a measure of QOL in AD involves a weighted average of patient and proxy reports. ${ }^{38}$ Future research should consider how patient and proxy information should be used for predicting outcomes and planning care.

\section{Limitations}

This analysis had a number of limitations including convenience sampling, lack of statistical power, relative sample homogeneity in terms of racial/ethnic status, and absence of potentially important proxy variables such as burden and distress. While we invited all patients to identify a proxy, only $25 \%$ of patients did so. Patients who did so were older, more cognitively impaired, and less functional in ADLs than patients who did not. Thus, we failed to capture the full range of cognitive and functional abilities, limiting our study's generalizability. We did not require that proxies be caregivers, and proxies who visited the participant once a week or less may not have had an accurate sense of the patient's cognition or functionality. However, most proxies lived with or frequently visited participants; the percentage of proxies who visited once a week or less was $<20 \%$. Some proxies failed to complete all three instruments, which could have introduced nonresponse bias, as it is possible that only the most dedicated proxies filled out all three instruments. Moreover, this study was limited in its assessment of applied cognition because of the exclusion of PROMIS-Cog from the follow-up analysis. Our conclusions about the preferability of patient versus proxy ratings were limited by this exclusion of PROMIS-Cog and the lack of gold-standard measures such as comprehensive neuropsychological testing.

\section{Conclusion}

Both patient and proxy input is important in the clinical assessment of a person with cognitive decline. Knowing in which instances patient and proxy reports are likely to differ is necessary to create an accurate clinical picture and guide treatment. Patient or proxy reports generally agree, and either might be relied upon with respect to functional status, though it may be preferable to weight proxy scores more heavily when assessing decline of IADLs over time. Patient and proxy reports differed on QOL related to executive function, a discrepancy that was correlated with lower depression levels and worse cognitive impairment. Though a patient's perspective on their own QOL may be preferred, further research is needed on combined patient-proxy measures, especially for QOL related to executive function. Patients and proxies also diverge in their assessment of applied cognition, and this discrepancy is associated with less depression and younger age. Combined measures may also be preferred for applied cognition measures. Importantly, further research is needed to assess the long-term predictive ability of patient and proxy measures and to validate these measures against "gold standards" such as comprehensive neuropsychological testing and direct observation in everyday functioning.

\section{Acknowledgment}

This study was supported by ARRA grant AG038825-01 from the National Institute on Aging (NIA) to Kathleen Smyth and Martha Sajatovic.

\section{Disclosure}

Dr Martha Sajatovic reports the following conflicts of interest: receiving research grants within the past three years from Merck, Alkermes, Janssen, Reuter Foundation, Woodruff Foundation, Reinberger Foundation, National Institutes of Health (NIH), Centers for Disease Control and Prevention (CDC); being a consultant for Bracket, Otsuka, Pfizer, Sunovion, Neurocrine, Supernus; royalties from Springer Press, Johns Hopkins University Press, Oxford Press, UpToDate, Lexicomp; and continuing medical education activities with American 
Physician Institute, MCM Education, and CMEology. The other authors report no conflicts of interest in this work.

\section{References}

1. Sands LP, Ferreira P, Stewart AL, Brod M, Yaffe K. What explains differences between dementia patients' and their caregivers' ratings of patients' quality of life? Am J Geriatr Psychiatry 2004;12(3):272-280.

2. Small GW, Rabins PV, Barry PP, et al. Diagnosis and treatment of Alzheimer disease and related disorders. Consensus statement of the American Association for Geriatric Psychiatry, the Alzheimer's Association, and the American Geriatrics Society. JAMA. 1997;278(16):1363-1371.

3. Daviglus ML, Bell CC, Berrettini W, et al. National Institutes of Health State-of-the-Science Conference statement: preventing Alzheimer's disease and cognitive decline. NIH Consens State Sci Statements. 2010; 27:1-30.

4. Martyr A, Clare L. Executive function and activities of daily living in Alzheimer's Disease: a correlational meta-analysis. Dement Geriatr Cogn Disord. 2010;33(2-3):189-203.

5. Loewenstein DA, Arguelles S, Bravo M, et al. Caregivers' judgments of the functional abilities of the Alzheimer's disease patient: a comparison of proxy reports and objective measures. J Gerontol B Psychol Sci Soc Sci. 2001;56(2):P78-P84.

6. Zanetti O, Geroldi C, Frisoni GB, Biachetti A, Trabucchi M. Contrasting results between caregiver's report and direct assessment of activities of daily living in patients affected by mild and very mild dementia: the contribution of the caregiver's personal characteristics. JAm Geriatr Soc. 1999;47(2):196-202.

7. Kiyak HA, Teri L, Borson S. Physical and functional health assessment in normal aging and in Alzheimer's disease: self-reports vs family reports. Gerontologist. 1994;34(3):324-330.

8. Magaziner J, Zimmerman SI, Gruber-Baldini AL, Hebel JR, Fox KM. Proxy reporting in five areas of functional status. Comparison with self-reports and observations of performance. Am J Epidemiol. 1997; 146(5):418-428

9. Ready RE, Ott BR, Grace J. Patient versus informant perspectives of quality of life in mild cognitive impairment and Alzheimer's disease. Int J Geriatr Psychiatry. 2004;19(3):256-265.

10. Wlodarczyk JH, Brodaty H, Hawthorne G. The relationship between quality of life, mini-mental state examination, and the instrumental activities of daily living in patients with Alzheimer's disease. Arch Gerontol Geriatr. 2004;39(1):25-33.

11. Vogel A, Mortensen EL, Hasselbalch SG, Andersen BB, Waldemar G. Patient versus informant reported quality of life in the earliest phases of Alzheimer's disease. Int J Geriatr Psychiatry. 2006;21(12):1132-1138.

12. Novella JL, Jochum C, Jolly D, et al. Agreement between patients' and proxies' reports of quality of life in Alzheimer's disease. Qual Life Res. 2001;10(5):443-452.

13. Tay L, Chua KC, Chan M, et al. Differential perceptions of quality of life (QoL) in community-dwelling persons with mild-to-moderate dementia. Int Psychogeriatr. 2014;26(8):1273-1282.

14. Ostbye T, Tyas S, McDowell I, Koval J. Reported activities of daily living: agreement between elderly subjects with and without dementia and their caregivers. Age Ageing. 1997;26(2):99-106.

15. Pol MC, Buurman BM, de Vos R, de Rooij SE. Patient and proxy rating agreements on activities of daily living and the instrumental activities of daily living of acutely hospitalized older adults. $J$ Am Geriatr Soc. 2011;59(8):1554-1556.

16. Huang HL, Chang MY, Tang JS, Chiu YC, Weng LC. Determinants of the discrepancy in patient- and caregiver-rated quality of life for persons with dementia. J Clin Nurs. 2009;18(22):3107-3118.

17. Galasko D, Bennett D, Sano M, et al. An inventory to assess activities of daily living for clinical trials in Alzheimer's disease. The Alzheimer's disease cooperative study. Alzheimer Dis Assoc Disord. 1997;11 (Suppl 2): S33-SS39.

18. Lai JS, Wagner LI, Jacobsen PB, Cella D. Self-reported cognitive concerns and abilities: two sides of one coin? Psychooncology. 2014; 23(10):1133-1141.
19. Becker H, Stuifbergen A, Lee H, Kullberg V. Reliability and validity of PROMIS cognitive abilities and cognitive concerns scales among people with multiple sclerosis. Int J MS Care. 2014;16(1):1-8.

20. National Institute of Neurological Disorders and Stroke (NINDS). User Manual for the Quality of Life in Neurological Disorders (Neuro-QoL) Measures; Version 2.0; March 2015. Available from: http://www. healthmeasures.net/images/neuro_qol/Neuro-QOL_User_Manual_ v2_24Mar2015.pdf. Accessed November 1, 2016.

21. Yesavage JA, Brink TL, Rose TL, et al. Development and validation of a geriatric depression screening scale: a preliminary report. J Psychiatr Res. 1982;17(1):37-49.

22. Parmelee PA, Lawton MP, Katz IR. Psychometric of the Geriatric Depression Scale among institutionalised aged. Psychol Assessment. 1989;1:331-338.

23. Tombaugh TN, McIntyre NJ. The mini-mental state examination: a comprehensive review. J Am Geriatr Soc. 1992;40(9):922-935.

24. Tariq SH, Tumosa N, Chibnall JT, Perry MH 3rd, Morley JE. Comparison of the Saint Louis University mental status examination and the mini-mental state examination for detecting dementia and mild neurocognitive disorder - a pilot study. Am J Geriatr Psychiatry. 2006; 14(11):900-910.

25. Rattanabannakit C, Risacher SL, Gao S, et al. The cognitive change index as a measure of self and informant perception of cognitive decline: relation to neuropsychological tests. J Alzheimers Dis. 2016;51(4):1145-1155.

26. McLoughlin DM, Cooney C, Holmes C, Levy R. Carer informants for dementia sufferers: carer awareness of cognitive impairment in an elderly community-resident sample. Age Ageing. 1996;25(5):367-371.

27. Gifford KA, Liu D, Hohman TJ, et al. A mutual self- and informantreport of cognitive complaint correlates with neuropathological outcomes in mild cognitive impairment. PLoS One. 2015;10(11):e0141831.

28. Gifford KA, Liu D, Carmona H, et al. Inclusion of an informant yields strong associations between cognitive complaint and longitudinal cognitive outcomes in non-demented elders. J Alzheimers Dis. 2015; 43(1):121-132.

29. Jonsson L, Andreasen N, Kilander L, et al. Patient- and proxy-reported utility in Alzheimer disease using the EuroQoL. Alzheimer Dis Assoc Disord. 2006;20(1):49-55.

30. Kozlowski AJ, Singh R, Victorson D, et al. Agreement between responses from community-dwelling persons with stroke and their proxies on the NIH neurological quality of life (Neuro-QoL) short forms. Arch Phys Med Rehabil. 2015;96(11):1986-1992.

31. Jacob L, Han JW, Kim TH, et al. How different are quality of life ratings for people with dementia reported by their family caregivers from those reported by the patients themselves? JAlzheimers Dis. 2017;55(1): 259-267.

32. Brod M, Stewart AL, Sands L, Walton P. Conceptualization and measurement of quality of life in dementia: the dementia quality of life instrument (DQoL). Gerontologist. 1999;39(1):25-35.

33. Bassett SS, Magaziner J, Hebel JR. Reliability of proxy response on mental health indices for aged, community-dwelling women. Psychol Aging. 1990;5(1):127-132.

34. Logsdon RG, Gibbons LE, McCurry SM, Teri L. Assessing quality of life in older adults with cognitive impairment. Psychosom Med. 2002; 64(3):510-519.

35. Zlatar ZZ, Moore RC, Palmer BW, Thompson WK, Jeste DV. Cognitive complaints correlate with depression rather than concurrent objective cognitive impairment in the successful aging evaluation baseline sample. J Geriatr Psychiatry Neurol. 2014;27(3):181-187.

36. Avondino E, Antione P. Heterogeneity of cognitive anosognosia and its variation with the severity of dementia in patients with Alzheimer's disease. J Alzheimers Dis. 2016;50(1):89-99.

37. Karlawish JH, Casarett D, Klocinski J, Clark CM. The relationship between caregivers' global ratings of Alzheimer's disease patients' quality of life, disease severity, and the caregiving experience. $J \mathrm{Am}$ Geriatr Soc. 2001;49(8):1066-1070.

38. Neumann PJ, Araki SS, Gutterman EM. The use of proxy respondents in studies of older adults: lessons, challenges, and opportunities. $J \mathrm{Am}$ Geriatr Soc. 2000;48(12):1646-1654. 


\section{Publish your work in this journal}

Patient Related Outcome Measures is an international, peer-reviewed, open access journal focusing on treatment outcomes specifically relevant to patients. All aspects of patient care are addressed within the journal and practitioners from all disciplines are invited to submit their work as well as healthcare researchers and patient support groups.

Submit your manuscript here: http://www.dovepress.com/patient-related-outcome-measures-journal

The journal is included in PubMed. The manuscript management system is completely online and includes a very quick and fair peer-review system. Visit http://www.dovepress.com/testimonials.php to read real quotes from published authors. 